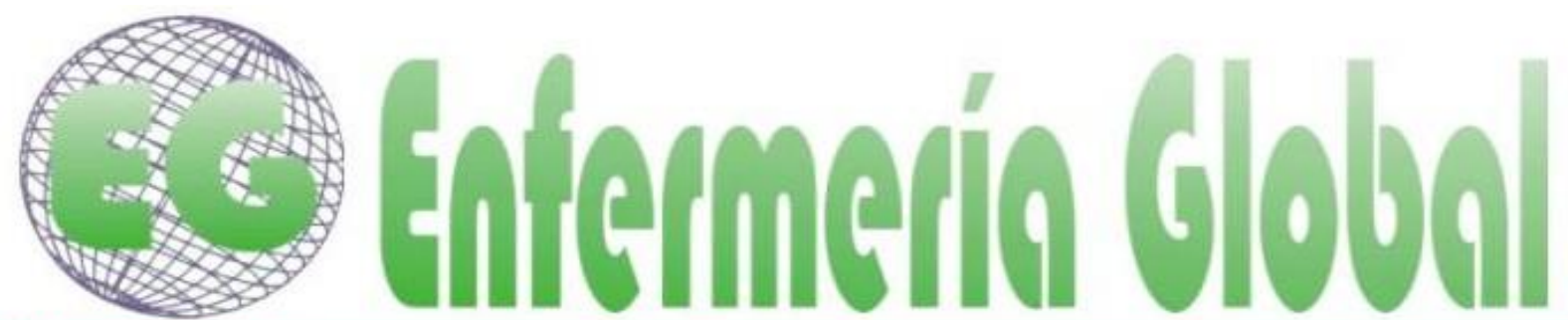

ISSN 1695-6141

$N^{\circ} 47$

Revista electrónica trimestral de Enfermería

Julio 2017

www.um.es/eglobal/

\title{
ORIGINALES
}

\section{Validación de la "Subjective Happiness Scale" en personas con Enfermedad Renal Crónica}

Validação da "Subjective Happiness Scale" em pessoas com Doença Renal Crónica Validation of the Subjective Happiness Scale in people with Chronic Kidney Disease

Luís Manuel Mota Sousa ${ }^{1}$

Cristina Maria Alves Marques-Vieira ${ }^{2}$

Sandy Silva Pedro Severino ${ }^{3}$

Juan Luis Pozo-Rosado ${ }^{4}$

Helena Maria Guerreiro José ${ }^{5}$

${ }^{1}$ Enfermero Especialista en Enfermería de Rehabilitación en el Hospital Curry Cabral, Centro Hospitalar Lisboa Central. Profesor de la Universidad Atlántica.Oeiras, Portugal.

${ }^{2}$ Profesora Asistente del Instituto de Ciencias de la Salud de la Universidad Católica Portuguesa.Portugal.

${ }^{3}$ Enfermera. Especialista en Enfermería de Rehabilitación del Hospital Curry Cabral,Portugal.

${ }^{4}$ Enfermero. Especialista en Enfermería de Rehabilitación del Hospital Fernando da Fonseca. Portugal.

${ }^{5}$ Enfermera Médico-Quirúrgica especialista, investigadora y estudiosa CIIS de la Academia Europea de Ciencias de Enfermería. Directora de la Escuela de Multipefil, Luanda, Angola Saúde.

E-mail: luismmsousa@gmail.com

http://dx.doi.org/10.6018/eglobal.16.3.266571

Recibido: $31 / 08 / 2016$

Aceptado: 02/10/2016

\section{RESUMEN:}

Objetivo: Verificar las propiedades psicométricas de la Subjective Happiness Scale (SHS), en personas con Enfermedad Renal Crónica (ERC), en hemodiálisis.

Método: Estudio metodológico cuya muestra aleatoria estuvo constituida por 171 personas con ERC que realizan hemodiálisis en dos clínicas de la región de Lisboa (Portugal). Los datos se obtuvieron entre Mayo y Junio del 2015. Las propiedades psicométricas estudiadas fueron la validez (constructo, convergente, discriminante), la fiabilidad (consistencia interna ( $\alpha$ de Cronbach)) y la estabilidad (Coeficiente de Correlación Intra-clase y de Correlación de Spearman-Brown).

Resultados: Los resultados confirman la estructura uni-factorial, presentando una fiabilidad con $\alpha=0,90$. La escala presenta una correlación positiva con la Escala de Satisfacción con la Vida $(r=0,60$; $\mathrm{p}<0,001$ ), lo que sirve para certificar su validez de criterio.

Conclusión: la versión portuguesa de la SHS es válida, fiable y reproducible en personas con ERC que realizan hemodiálisis.

Palabras clave: Insuficiencia Renal Crónica; Estudios de Validación; Psicometría; Felicidad; Enfermería. 


\title{
RESUMO:
}

Objetivo: Validar as propriedades psicométricas da Subjective Happiness Scale (SHS) em pessoas com Doença Renal Crônica (DRC) em programa de hemodiálise.

Método: Trata-se de um estudo metodológico. A amostra randomizada foi constituída por 171 pessoas com DRC submetida a hemodiálise em duas clinicas na região de Lisboa, Portugal. Os dados foram colhidos de maio a junho de 2015. Foram avaliadas as propriedades psicométricas: validade (construto, convergente e discriminativa), fidedignidade por meio da consistência interna ( $\alpha$ de Cronbach) e estabilidade (Coeficiente de Correlação Intraclasse e Coeficiente de Correlação de Spearman-Brown).

Resultados: Os resultados suportam a estrutura unifatorial, com uma confiabilidade $(\alpha=0,90)$. Além disso, esta escala está positivamente correlacionada com a Escala de Satisfação com a Vida $(r=0,60$; $\mathrm{p}<0,001$ ), apoiando assim a sua validade de critério.

Conclusões: A versão portuguesa da SHS é válida e reprodutível em pessoas com DRC.

Palavras chaves: Insuficiência Renal Crónica; Estudos de Validação; Psicometria; Felicidade; Enfermagem.

\begin{abstract}
:
Objective: To explore the psychometric properties of the Subjective Happiness Scale (SHS) in patients with Chronic Kidney Disease (CKD) under hemodialysis.

Methods: This is a methodological study. The random sample included 171 patients with CKD under hemodialysis program in two clinics in the region of Lisbon, Portugal. Data was collected between May and June 2015. The following psychometric properties were evaluated: validity (construct, convergent and discriminant), reliability through internal consistency (Cronbach $\alpha$ ) and stability (intraclass correlation coefficient and Spearman-Brown correlation coefficient).

Results: The results support the unifactorial structure, with reliability $(\alpha=0.90)$. In addition, this scale is positively correlated with the Satisfaction Life Scale $(r=0.60 ; p<0.001)$, supporting the validity criteria.

Conclusions: The Portuguese version of the SHS is valid and reproducible in patients with CKD.
\end{abstract}

Keywords: Renal Insufficiency; Chronic; Validation studies; Psychometrics; Happiness; Nursing.

\section{INTRODUCCIÓN}

La Enfermedad Renal Crónica (ERC) es una enfermedad degenerativa, caracterizada por la destrucción progresiva, gradual e irreversible de un gran número de nefronas y que tiene como consecuencia una disminución de la capacidad renal de excretar metabolitos, o sea, una pérdida de la función renal ${ }^{(1-4)}$. La hemodiálisis representa una de las opciones terapéuticas para el tratamiento de las personas con ERC. Esta técnica consiste en la filtración extra corporal de la sangre mediante la utilización de una máquina de hemodiálisis. La realización de hemodiálisis provoca un impacto sobre diferentes áreas del individuo, área física, psicológica, social, así como en su vida personal y familiar ${ }^{(1)}$.

El bienestar subjetivo o felicidad ${ }^{(5)}$ puede ser definido como un estado de extroversión estable, que parece estar relacionado con la facilidad de la persona para socializar, pudiendo favorecer una interacción natural y placentera con las otras personas. La persona con un nivel de bienestar elevado parece tener mejores relaciones sociales que las personas con un nivel de bienestar más bajo ${ }^{(5)}$.

La Subjective Happiness Scale (SHS), desarrollada por Lyubomirski y Lepper ${ }^{(6)}$ está compuesta por cuatro elementos a través de los cuales se solicita a los participantes, mediante dos tipos de afirmaciones, que se caractericen a sí mismos en comparación con sus semejantes, tanto de una forma absoluta, como de una forma relativa (elementos dos y tres). Por otro lado, los elementos uno y cuatro de la escala, corresponden a la descripción del autoconcepto de felicidad e infelicidad. En el último elemento de la escala la puntuación obtenida se invierte. 
En esta escala se solicita a los participantes para cuantificar en grados si se identifican con las afirmaciones incluidas. Las respuestas se clasifican atendiendo a una escala visual analógica con siete grados, que describen dos afirmaciones antagónicas para expresar el nivel de felicidad o su ausencia. Los autores construyeron la medida unidimensional de la escala, con cuatro apartados, para evitar la sobrecarga de los participantes.

La SHS fue desarrollada para la población norteamericana y rusa ${ }^{(6)}$, a pesar de ello, ya fue validada para otras poblaciones, como la japonesa ${ }^{(7)}$, la china, la malaya ${ }^{(8)}$, la australiana, la alemana, la filipina ${ }^{(9)}$, los estudiantes chinos ${ }^{(10)}$, la población árabe ${ }^{(11)}$, la española ${ }^{(12)}$, la italiana ${ }^{(13)}$, para el portugués europeo ${ }^{(14-15)}$, para el portugués de Brasil $^{(16)}$ y para la población mexicana ${ }^{(17)}$.

La validez de constructo solo se demostró en una dimensión. Se realizó un análisis factorial exploratorio ${ }^{(6,9-10,12-16)}$, donde se analizaron los Componentes Principales ${ }^{(12)} 0$ Principal Axis Factoring ${ }^{(9,16)}$ con rotación quartimax ${ }^{(9)}$ y también un análisis factorial confirmatorio $^{(10-17)}$.

La validez concurrente se comprobó mediante la utilización de otras medidas relacionadas con la felicidad $^{(6,9)}$, la satisfacción con la vida ${ }^{(9,13-16)}$, la afectividad $^{(6,9)}$, la autoestima $^{(6,16)}$, la esperanza ${ }^{(16)}$, la calidad de vida ${ }^{(10)}$, la depresión ${ }^{(6,13)}$, y la ansiedad $^{(13-14)}$.

En relación a la confiabilidad de la escala original, esta presenta un a de Cronbach que varía entre 0,79 y 0,94 $(\mathrm{M}=0,86)$, lo que demuestra una buena consistencia interna. También muestra estabilidad en el tiempo, con una confiabilidad del test retest que varía entre 0,55 y $0,90(\mathrm{M}=072)^{(6)}$. La escala revela en todas las poblaciones, ser un instrumento válido y confiable para evaluar la felicidad subjetiva ${ }^{(7-}$ 16). La versión portuguesa de la escala evidencia propiedades psicométricas idénticas a las de la versión original ${ }^{(6)}$ y puede ser utilizada para realizar comparaciones en investigaciones transculturales ${ }^{(15)}$.

De esta forma, este estudio pretende verificar si la versión portuguesa de la $\mathrm{SHS}^{(15)}$ es válida y confiable cuando utilizada en personas con ERC que realizan programas de hemodiálisis.

\section{METODOLOGÍA}

El estudio realizado tiene carácter metodológico ${ }^{(18)}$. Se realizó en dos unidades de diálisis de la Clínica Diaverum, en la región de Lisboa, de mayo a junio del año 2015. La muestra fue constituida por personas con ERC en programa de hemodiálisis.

Los criterios de inclusión utilizados fueron: personas con ERC que realizan tratamiento de hemodiálisis hace, por lo menos, seis meses, con edad igual o superior a 18 años. Fueron excluidas las personas con déficit cognitivo y/o enfermedad psiquiátrica activa. La información acerca de estos criterios de exclusión se obtuvo a través de la historia clínica del paciente.

La población comprende un grupo de 248 personas con ERC, que cumplieron los criterios de inclusión (139 procedentes de la Clínica 1 y 114 de la Clínica 2). De esta manera la muestra fue compuesta por las personas que cumplieron los criterios de elegibilidad y su selección fue realizada siguiendo un método probabilístico (con aleatoriedad sin reposición, con un intervalo de confianza del $95 \%$ y un error de la 
muestra del $5 \%)^{(19)}$, para una muestra mínima necesaria de 192 personas (103 en la clínica 1 y 89 en la clínica 2). Posteriormente a esta fase se realizó una selección aleatoria sin reposición. Del grupo de personas seleccionadas en la clínica 1, 6 personas rechazaron participar, 2 fueron ingresadas y 2 desistieron. Ya en la clínica 2, 4 personas rechazaron participar, 2 fueron ingresadas y 5 desistieron. En total se obtuvieron los datos de 171 personas, 93 de la clínica 1 (89\%) y 78 (88\%) de la clínica 2.

Para realizar la recogida de los datos se utilizó la versión portuguesa de la $\mathrm{SHS}^{(15)}$ en la evaluación de la felicidad y para la evaluación de la satisfacción con la vida en general fue utilizado el Índice de Bienestar personal (IBP), cuya versión original es el Personal Well being Índex (PWI), desarrollado a partir de la Comprehensive Quality of Life Scale ${ }^{(21)}$. También fue utilizado un instrumento para caracterizar la muestra a nivel sociodemográfico y clínico, más específicamente, su edad, género, nacionalidad, escolaridad, actividad profesional, estado civil, tiempo de diálisis, presencia de Hipertensión Arterial y de Diabetes.

En la SHS se solicita al encuestado que cuantifique el grado en que se identifica con las cuatro afirmaciones existentes, utilizando como referencia una escala visual analógica graduada de 0 a 7 . La versión portuguesa presenta un único factor y su fiabilidad interna representado por el a de Cronbach es de $0,76^{(15)}$.

EI IBP está constituido por siete dominios (satisfacción con el nivel de vida, salud, realización personal, relaciones personales, sentimiento de seguridad, conexión con la comunidad y seguridad en el futuro) que pretenden evaluar la "satisfacción con la vida en general". En cada pregunta se solicita a los participantes para determinar el valor que atribuyen a su grado de satisfacción en cada dominio, en una escala de "0" (extremamente insatisfecho) a "10" (extremamente satisfecho), con una posición intermedia neutra. El IBP se calcula a través del contaje de la puntuación obtenida comprendida entre 0-100 (porcentaje máximo de la escala, \%SM). El análisis factorial confirmatorio de la versión portuguesa demuestra la existencia de un único factor, con un valor de Alpha de Cronbach de 0,81(20).

Los análisis estadísticos se realizaron a través de la utilización del Statistical Package for Social Sciences (SPSS) versión 20.0. La reproductibilidad fue estudiada a través de la confiabilidad interna y la estabilidad. El estudio de la fiabilidad, realizado para la evaluación de las propiedades psicométricas, se materializó a través del cálculo del a de Cronbach e para evaluar la estabilidad fue utilizado el Coeficiente de Correlación Intra-clase (CCl) y el Coeficiente de Correlación de Spearman-Brown ${ }^{(22)}$ en el Test Retest (48 a 96 horas después, en 40 personas seleccionadas aleatoriamente; 26 por cuestionario y 14 por entrevista). Como valor mínimo satisfactorio se determinó un valor de consistencia interna de $0,70^{(22)}$. En este estudio se analizó la validez de constructo, concurrente y discriminante. La validez de constructo se obtuvo mediante el Análisis factorial exploratorio (AFE) y Análisis factorial confirmatorio (AFC). EI AFE se realizó utilizando el método de la máxima verosimilitud, con rotación Varimax. La idoneidad fue estimada a través del Kaiser-Meyer-Olkin (KMO) y el test de esfericidad de Bartlett.

El AFC se realizó mediante la utilización del software AMOS 21.0. Se utilizó el método robusto de estimación con máxima verosimilitud ${ }^{(23)}$. Los índices de ajuste utilizados para comprobar el ajuste del modelo fueron: relación entre el Qui cuadrado y los 
grados de libertad (X2/g.I); goodness-of-fit index (GFI), root mean square error of approximation (RMSEA); comparative fit index (CFI), Tucker-Lewis index (TLI) ${ }^{22-23)}$.

Estos índices pueden ser clasificados en índices absolutos e índices relativos. Los Índices Absolutos evalúan la calidad del modelo, sin comparación con otros modelos. Los índices más utilizados en esta família son: X2/g.I., cuando X2/g.I=1, el ajuste ajuste es perfecto, cuando X2/g.I es inferior a 2 , el ajuste es bueno, es aceptable cuando X2/g.I < a 5, e inaceptable cuando X2/g.I > a 5. El Root Mean Square Residual (RMSR) se determina dividendo la raíz cuadrada de la matriz de los errores, entre los grados de libertad. Así, cuanto menor es el RMSR mejor será el ajuste. Cuando $\mathrm{RMSR}=0$ indica que el ajuste es perfecto. El Goodness of Fit Index (GFI) explica la proporción de la co-varianza observada entre las variables presentes, explicadas por los modelos ajustados. De forma general, se considera que GFI < a 0.8 indica modelos mal ajustados a los datos; GFI entre [0.9; 0.95[ indica un buen ajuste; GFI > a 0.95 indica ajuste muy bueno y $\mathrm{GFI}=1$ ajuste perfecto. Los Índices Relativos evalúan la calidad del modelo en test, en relación al modelo con menor ajuste posible y/o al modelo com el mejor ajuste posible. El Comparative Fit Index (CFI) compara el ajuste del modelo en estudio (X2) con los Grados de libertad (gl), con el ajuste del modelo basal con los grados de libertad. Generalmente se acepta que CFI < a 0.9 indica modelos mal ajustados; CFI entre [0.9; 0.95[ indica un buen ajuste; CFI > a 0.95 ajuste muy bueno y $\mathrm{CFI}=1$ ajuste perfecto. El Tucker-Lewis Index (TLI), también conocido por Bentler-Bonett-non-normed fit index (NNFI), en que los valores del TLI varían entre 0 y 1 . Los valores próximos de 1 indican ajuste muy bueno ${ }^{(23)}$.

La validez convergente se comprobó mediante el Coeficiente de Correlación de Pearson entre la SHS y el IBP. Para verificar la validez discriminante fue utilizado el test $t$ Student para muestras independientes y la ANOVA para más de dos muestras independientes (fue confirmada la distribución normal a través del test de KS de Kolmogorov-Smirnov).

Las variables categóricas se expresaron en porcentaje o valor absoluto y las continuas como media \pm desvío estándar o mediana. El punto de corte utilizado fue la mediana en la edad y en el tiempo de tratamiento de hemodiálisis, para crear variables de categoría, con un nivel de significancia adoptado de $p<0,05$.

Se solicitó autorización al autor de la versión portuguesa de la $\mathrm{SHS}^{(15)}$ y esta fue concedida.

Este estudio fue aprobado por la Comisión de Ética de Diaverum ( $\mathrm{n}$ 1/2015). Todos los participantes firmaron un documento de consentimiento libre y esclarecido después de informados sobre la garantía del sigilo de sus datos personales y su derecho de renuncia.

\section{RESULTADOS}

El perfil sociodemográfico y sanitario de los participantes está expuesto en la tabla 1. En el presente estudio la media de edad de la muestra es de 60,20 $\pm 14,34$ años. La mayoría son hombres $(61 \%)$, de nacionalidad portuguesa $(80,1 \%)$, con 4 años de escolaridad (42,9\%), jubilados $(76,7 \%)$ y que están casados $(56,5 \%)$. En lo referente a los datos sanitarios, los sujetos de la muestra realizan hemodiálisis hace $72,17 \pm 54,23$ meses, el 62,1\% presentan Hipertensión Arterial y el 27,1\% Diabetes. 
Tabla 1 - Características de las personas con ECR evaluadas en las dos clínicas de hemodiálisis. Lisboa, Portugal, 2015.

\begin{tabular}{|c|c|c|}
\hline 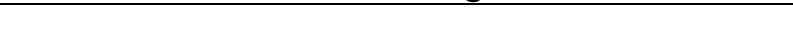 & $\mathrm{F}$ & $\%$ \\
\hline Género & & \\
\hline Femenino & 66 & 38,6 \\
\hline Masculino & 105 & 61,4 \\
\hline \multicolumn{3}{|l|}{ Nacionalidad } \\
\hline Portuguesa & 137 & 80,1 \\
\hline Caboverdiana (Cabo Verde) & 24 & 14,0 \\
\hline Santotomense (Santo Tomé y Príncipe) & 6 & 3,5 \\
\hline Guineana (Guinea Bissau) & 1 & 0,6 \\
\hline Angolana (Angola) & 3 & 1,8 \\
\hline \multicolumn{3}{|l|}{ Escolaridad } \\
\hline Analfabeto & 6 & 3,6 \\
\hline 4 Año de Educación Primaria Obligatoria & 72 & 42,9 \\
\hline $\begin{array}{l}\text { 6 Año de Educación Primaria Obligatoria } \\
\text { 9o Año de Educación Secundaria }\end{array}$ & $\begin{array}{l}31 \\
25\end{array}$ & $\begin{array}{l}18,5 \\
14,9\end{array}$ \\
\hline 12º Año de Educación Secundaria & 19 & 11,3 \\
\hline Licenciatura & 13 & 7,7 \\
\hline Máster/Doctorado & 2 & 1,2 \\
\hline \multicolumn{3}{|l|}{ Actividad profesional } \\
\hline Jubilado & 125 & 76,7 \\
\hline Activo & 38 & 23,3 \\
\hline \multicolumn{3}{|l|}{ Estado civil } \\
\hline Soltero (a) & 44 & 25,9 \\
\hline Casado (a) & 96 & 56,5 \\
\hline Viudo (a) & 20 & 11,8 \\
\hline Divorciado/separado & 10 & 5,9 \\
\hline \multicolumn{3}{|l|}{ Hipertensión Arterial } \\
\hline No & 64 & 37,9 \\
\hline Sí & 105 & 62,1 \\
\hline \multicolumn{3}{|l|}{ Diabetes } \\
\hline No & 124 & 72,9 \\
\hline Sí & 46 & 27,1 \\
\hline
\end{tabular}

\section{Confiabilidad y Estabilidad}

En el análisis de las propiedades psicométricas, la reproductibilidad de la SHS se verificó a través del Coeficiente de a de Cronbach, presentando un valor que varió de 
0,85 a 0,90, después de excluir cada una de las dimensiones. En relación con la estabilidad (Test Retest), los datos que se obtuvieron a través del cuestionario $(n=26)$, presentaron un a de Cronbach global en la primera evaluación de 0,80 y de 0,61 en la segunda, así mismo, el valor del Coeficiente de Correlación de Spearman-Brown fue de 0,82 y el CCI de 0,82 [IC95\%; 0,71 a 0,91, p<0,001]. Los datos obtenidos mediante la entrevista $(n=14)$ presentaron un a de Cronbach global en la primera evaluación de 0,83 y de 0,85 en la segunda, con un Coeficiente de Correlación de Spearman-Brown de 0,77 y un $\mathrm{CCl}$ de 0,87 [IC95\%; 0,74 a 0,95, $\mathrm{p}<0,001]$. De esta manera, se demostró la consistencia y estabilidad entre evaluaciones de la SHS, tanto por medio de cuestionario como de entrevista.

\section{Validez}

El análisis factorial exploratorio $(\mathrm{KMO}=0,83$; test de esfericidad de Bartlett $\mathrm{X} 2$ [6] $486,679, p<0,001)$ presentó una única solución factorial, que fue responsable por el $72,10 \%$ de la varianza explicada del constructo. Todas las dimensiones fueron cargadas en el factor, con cargas factoriales adecuadas, o sea >0,6 (ver Tabla 2). El valor del Coeficiente a de Cronbach fue de 0,90.

Tabla 2 - Análisis factorial exploratorio de la versión portuguesa de la SHS en personas con ERC. Lisboa, Portugal, 2015.

\begin{tabular}{l|c}
\hline & $\begin{array}{c}\text { Factor } 1 \\
\text { FS }^{*}\end{array}$ \\
\hline SHS1- En general, me considero & 0,912 \\
SHS2- En comparación con las otras personas como yo, me & \\
considero & 0,909 \\
SHS3- Algunas personas son generalmente muy felices. & 0,868 \\
SHS4- Algunas personas generalmente no son muy felices & 0,687 \\
\hline Números propios (Eigenvalue) & 3,142 \\
Varianza explicada & 72,10 \\
Coeficiente alfa & 0,90 \\
Média (DP) & 19,97 \\
& $( \pm 5,96)$ \\
\hline
\end{tabular}

${ }^{*}$ Felicidad Subjetiva

En el análisis factorial confirmatorio se obtuvo X2/g.I=0,971, RMSEA=0,00; $\mathrm{GFI}=0,994, \mathrm{CFI}=1,00$ y $\mathrm{TLI}=1,00$. Estos índices presentan un ajuste muy bueno para la hipótesis del solución de un factor (figura 1).

Figura 1 - Modelo de Análisis factorial confirmatorio de la SHS

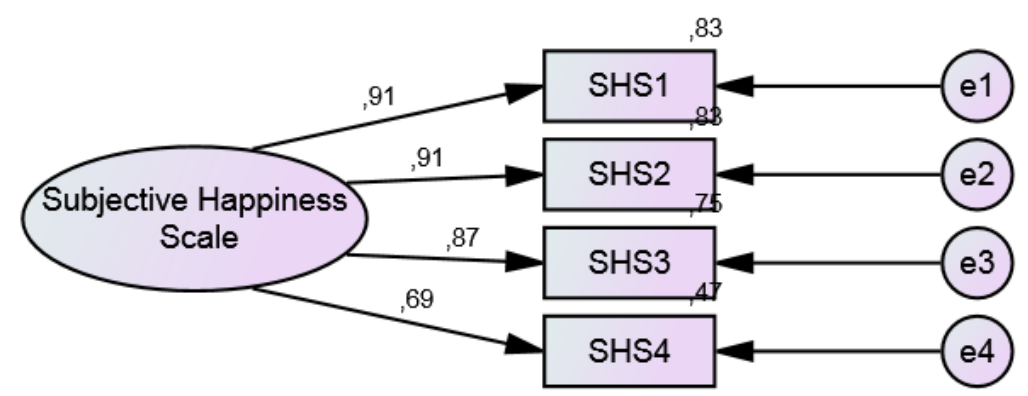


En el estudio de la validez convergente, se confirma una correlación positiva moderada $(r=0,60 ; \mathrm{p}<0,001)$ entre la puntuación de la SHS y la del IBP.

En la tabla 3 se observan los resultados referentes a la validez discriminante, que muestran si la SHS es capaz de diferenciar la felicidad subjetiva en las variables género, edad, nacionalidad, escolaridad, actividad profesional, estado civil, presencia de Hipertensión Arterial, Diabetes y tiempo de tratamiento de diálisis.

La SHS consigue discriminar la felicidad subjetiva según la edad, nacionalidad, actividad profesional y presencia de Diabetes. De este modo, las personas con edad inferior a 63 años y con actividad profesional, presentan niveles de felicidad superiores. Ya las personas de nacionalidad portuguesa, presentan niveles de felicidad inferiores a las de nacionalidad de un país africano. Además, las personas con Diabetes presentan niveles de felicidad inferiores a las que no tienen Diabetes.

Tabla 3 -Validez discriminante de la SHS. Lisboa, Portugal, 2015.

\begin{tabular}{l|l|l}
\hline Género & Média $( \pm \mathrm{DP})$ & $p$-value \\
Masculino & $19,8 \pm 5,6$ & 0,630 \\
Femenino & $20,2 \pm 6,4$ & \\
\hline Edad & $21,3 \pm 5,9$ & 0,006 \\
Inferior a 63 años & $18,8 \pm 5,7$ & \\
Superior a 63 años & $19,4 \pm 6,1$ & 0,020 \\
\hline Nacionalidad & $22,1 \pm 5,2$ & \\
Portuguesa & & \\
Otra & $19,7 \pm 5,9$ & 0,150 \\
\hline Escolaridad & $21,3 \pm 5,4$ & \\
Inferior a 12 años & & \\
Superior a 12 años & $19,3 \pm 5,9$ & 0,010 \\
\hline Actividad profesional & $22,1 \pm 5,6$ & \\
Jubilado & $20,6 \pm 5,7$ & 0,151 \\
Activo & $20,2 \pm 6,1$ & \\
\hline Estado civil & $18,1 \pm 5,9$ & \\
Soltero (a) & & \\
Casado (a) & $20,6 \pm 5,6$ & 0,280 \\
Otro & $19,6 \pm 6,1$ & \\
\hline Hipertensión Arterial & $20,6 \pm 5,8$ & 0,018 \\
No & & \\
Sí & Diabetes & \\
No & & \\
\hline
\end{tabular}




\begin{tabular}{l|l|l} 
Sí & $18,2 \pm 6,2$ & \\
\hline Tiempo de hemodiálisis & & \\
Inferior a 60 meses & $19,5 \pm 5,6$ & 0,360 \\
Superior a 60 meses & $20,3 \pm 6,3$ & \\
\hline
\end{tabular}

\section{DISCUSION}

Los valores de confiabilidad fueron semejantes a los obtenidos con la versión original de los Estados Unidos ${ }^{(6)}$ y superiores a los de las versiones austriaca $(\alpha=, 080)^{(9)}$, filipina $(\alpha=, 082)^{(9)}$, china $(\alpha=, 082)^{(10)}$, libanesa $(\alpha=, 074)^{(12)}$, española $(\alpha=, 081)^{(13)}$, italiana $(\alpha=, 079)^{(14)}$, portugués europeo $(\alpha=, 077)^{(15)}$ y de Brasil $(\alpha=, 084)^{(16)}$. Los valores del $\alpha$ de Cronbach obtenidos en este estudio son considerados buenos ${ }^{(22)}$.

En relación a la estabilidad, el valor de las correlaciones, tanto en el cuestionario como en la entrevista fue superior al estudio original $(r=0,55 \text { a } 0,86)^{(6)}$, a la versión china $(r=0,70)^{(10)}$ y a la versión española $(r=0,72)^{(13)}$. En este estudio los valores son superiores a 0,7 , lo que indica que existe una buena estabilidad de la medida ${ }^{(22)}$. Se obtuvo soporte para la estabilidad de esta medida en la modalidad de cuestionario y entrevista.

Todas las dimensiones fueron cargadas en un único factor, a semejanza de otros estudios que realizaron el análisis factorial exploratorio(6-7,9-10,16). El peso factorial fue idéntico a las versiones china, portugués de Brasil $(>0,60)$ y superior a la versión libanesa $(>0,46)$. El valor de $\mathrm{KMO}$ fue superior a las versiones austríaca $(\mathrm{KMO}=0,80)^{(9)}$, filipina $(\mathrm{KMO}=0,65)^{(9)}$, china $(\mathrm{KMO}=0,79)^{(10)}$, libanesa $(\mathrm{KMO}=0,75)^{(12)} \mathrm{y}$ portugués de Brasil $(\mathrm{KMO}=0,79)^{(16)}$. En este estudio el valor de $\mathrm{KMO}$ es bueno y revela que el modelo se adapta a los datos ${ }^{(20)}$. La varianza explicada para un factor fue superior a las versiones austríaca $(65,7 \%)^{(9)}$, filipina $(53,2 \%)^{(9)}$, china $(65,3 \%)^{(10)}$, libanesa $(45,2 \%)^{(12)}$ y portugués de Brasil $(64,2 \%)^{(16)}$. De esta forma, se demuestra que la puntuación de la SHS es válida para medir la felicidad subjetiva. En este estudio el AFC también confirma la solución de un factor ${ }^{(11-17)}$. La validez convergente presentó resultados en el sentido esperado, es decir, que las puntuaciones altas de la SHS están asociadas a puntuaciones altas del IBP (satisfacción con la vida en general). Se obtuvo confirmación para la validez externa de la medida, a semejanza de las muestras ${ }^{(9,13-16)}$.

La SHS cuando aplicada en personas con ERC en programa de hemodiálisis consigue discriminar según la edad, situación profesional, nacionalidad y presencia de Diabetes. En la versión libanesa se comprueba que esta medida consigue discriminar según la edad y si hubo rechazo de los padres en la infancia ${ }^{(12)}$. En la versión italiana se observa que los hombres de edad tienen un porcentaje menor de puntuaciones altas (efecto techo) y un mayor porcentaje de puntuaciones bajas (efecto suelo) cuando comparados con las mujeres y los jóvenes ${ }^{(14)}$.

Las principales limitaciones se relacionan con la falta de financiación y el tamaño de la muestra.

Los resultados de este estudio están en concordancia con la escala original y con todas las otras versiones adaptadas de la $\mathrm{SHS}^{(7-16)}$. Sugieren que la versión en portugués europeo de la SHS es una medida válida y confiable para evaluar la felicidad subjetiva en personas con ERC, en formato de cuestionario y/o entrevista. 
Para este estudio fue utilizada una muestra representativa de personas con ERC en programa de diálisis. Sin embargo, en futuras investigaciones puede ser interesante realizar un análisis factorial confirmatorio para esta población específica con una muestra superior a 300 personas.

\section{CONCLUSIONES}

La SHS utilizada en personas con ERC, es un instrumento fiable, reproducible y válido cuando se aplica en el formato de cuestionario y/o entrevista. Esta escala presenta buenas propiedades psicométricas, permitiendo así decir que puede ser considerada un buen instrumento de medida, con propiedades semejantes entre la versión original, la portuguesa y las versiones en otros idiomas y culturas.

\section{REFERENCIAS}

1. Takemoto AY, Okubo P, Bedendo J, Carreira L. Avaliação da qualidade de vida em idosos submetidos ao tratamento hemodialítico. Rev Gaúcha Enferm., Porto Alegre (RS) 2011 jun;32(2):256-62.

2. Oliveira CS, da Silva EC, Ferreira LW, Skalinski L.M. Perfil dos pacientes renais crônicos em tratamento hemodialítico. Rev Baiana Enferm. 2015; 29(1):42-49.

3. Ottaviani AC, Souza EN, Drago NC, de Mendiondo MSZ, PavariniSCL, Orlandi FS. Esperança e espiritualidade de pacientes renais crônicos em hemodiálise: estudo correlacional. RevLatino-AmEnferm. 2014;22(2):248-54.

4. Bosenbecker NRV, Menegon MBC, Zillmer JGV, Dall'agnol J. Perfil das pessoas em hemodiálise de um serviço de nefrologia. J Nurs Health. 2015;5(1):38-46.

5. Passareli PM, Silva JAD. Psicologia positiva e o estudo do bem-estar subjetivo. EstudPsicol (Campinas). 2007; 24(4): 513-517.

6. Lyubomirsky S, Lepper HS. A measure of subjective happiness: preliminary reliability and construct validation. Soc Indic Res.1999;46(2):137-55.

7. Shimai S, Otake K, Utsuki N, Ikemi A, Lyubomirsky S. Development of a Japanese version of the Subjective Happiness Scale (SHS), and examination of its validity and reliability. Nippon Koshu Eisei Zasshi. 2004; 51: 845-853.

8. Swami V. Translation and validation of the Malay Subjective Happiness Scale. Soc Indic Res. 2008; 88: 347-353.

9. Swami V, Stieger S, Voracek M, Dressler SG, Eisma L, Furnham A. (2009). Psychometric evaluation of the Tagalog and German Subjective Happiness Scale and a cross-cultural comparison. Soc Indic Res. 2009;93:393-406.

10. Nan H, Ni MY, Lee PH, Tam WW, Lam TH, Leung GM, McDowell I. Psychometric evaluation of the Chinese version of the subjective happiness scale: evidence from the Hong Kong FAMILY Cohort. Int J Behav Med. 2014; 21(4): 646-652.

11. Moghnie L, Kazarian SS. Subjective happiness of Lebanese college youth in Lebanon: Factorial structure and invariance of the Arabic Subjective Happiness Scale. Soc Indic Res. 2012; 109(2): 203-210.

12. Extremera N,Fernández-Berrocal P. The Subjective Happiness Scale: Translation and preliminary psychometric evaluation of a Spanish version. Soc Indic Res. 2014;119(1): 473-481.

13. Iani L, Lauriola M, Layous K, Sirigatti S. Happiness in Italy: translation, factorial structure and norming of the subjective happiness scale in a large community sample. Soc Indic Res. 2014; 118(3): 953-967.

14. Spagnoli $\mathrm{P}$, Caetano A, Silva A. Psychometric properties of a Portuguese version of the Subjective Happiness Scale. Soc Indic Res. 2012;105(1): 137-143. 
15. Pais-Ribeiro JL. Validação transcultural da Escala de Felicidade Subjectiva de Lyubomirsky e Lepper. Psicol Saúde Doenças. 2012; 13(2): 157-168.

16. Damásio BF, Zanon C, Roller, SH. Validation and psychometric properties of the Brazilian version of the Subjective Happiness Scale. Univ Psychol. 2014; 13(1): 1724.

17. Quezada L, Landero R, González MT. A validity and reliability study of the Subjective Happiness Scale in Mexico. J Happiness Well-Being. 2016; 4(1), 90100.

18. De Lima DVM. Desenhos de pesquisa: uma contribuição para autores. Online Braz J Nurs (Online). 2011[Acceso 18 Agosto 2015]; 10(2). Disponible en:http://www.objnursing.uff.br/index.php/nursing/article/view/3648/html

19. Santos GEO. Cálculo amostral: calculadora on-line. [Acceso 1 maio 2015].Disponible en: http://www.calculoamostral.vai.la.

20. Sousa LM, Marques-Vieira CM, Severino SS, Pozo-Rosado JL, José HM. Validación del Índice de Bien-estar Personal en personas con enfermedad renal crónica. Enfermería Nefrológica. 2016;19(2):135-41.

21. Cummins RA, McCabe MP, Romeo Y, Gullone E. The Comprehensive Quality of Life Scale: Instrument development and psychometric evaluation on tertiary staff and students. Educ. Psychol. Measur. 1994; 54: 372-382.

22. Sousa LMM, Marques-Vieira CMA, Carvalho ML, Veludo F, José, HMG. Fidelidade e validade na construção e adequação de instrumentos de medida. Enformação. 2015[Acceso 17 agosto 2015]; 5:25-32. Disponible en: http://www.acenfermeiros.pt/index.php?id1=15\&id2=9.

23. Marôco J. Análise de equações estruturais: Fundamentos teóricos, software \& aplicações. 2.ed. Pero Pinheiro: ReportNumber, Lda; 2014.

ISSN 1695-6141

๑ COPYRIGHT Servicio de Publicaciones - Universidad de Murcia 